\title{
Protée
}

\section{Le monde naturel, entre corps et cultures}

\section{Gianfranco Marrone}

Volume 34, numéro 1, printemps 2006

Fortune et actualité de $D u$ sens

URI : https://id.erudit.org/iderudit/013309ar

DOI : https://doi.org/10.7202/013309ar

Aller au sommaire du numéro

Éditeur(s)

Département des arts et lettres - Université du Québec à Chicoutimi

ISSN

0300-3523 (imprimé)

1708-2307 (numérique)

Découvrir la revue

Citer cet article

Marrone, G. (2006). Le monde naturel, entre corps et cultures. Protée, 34(1), 47-55. https://doi.org/10.7202/013309ar

\section{Résumé de l'article}

À partir d'un projet de recherche sur la gestualité (presque abandonné ensuite par la sémiotique structurale), Greimas avait élaboré dans $\mathrm{Du}$ sens la notion de " monde naturel ", en parallèle à celle de " langue naturelle ". La relation entre mots et choses n'est pas référentielle (de représentation) mais intersémiotique (de traduction). Cette idée pose les bases théoriques d'une sémiotique où le sens est vu comme possibilité virtuelle de transcodage et la signification comme réalisation concrète de ce même transcodage. Relire les pages greimassiennes dédiées à ces questions permet aussi d'interroger certaines hypothèses actuelles de naturalisation du sens et du corps. 


\section{LE MO N DE N ATU REL, ENTRE CO RPS ET CU LTU RES}

GIANFRANCO MARRONE

Selon Greimas, pour expliquer la signification et mieux comprendre le sens des textes, il faut les lire à partir de la fin. Il en va ainsi pour un récit mythique bororo (Greimas, 1970: 185-230), pour un conte littéraire comme «Deux amis» de Maupassant (Greimas, 1976) ou pour un livre scientifique comme les Prolégomènes de Hjelmslev (Greimas, 1970: 93-94). De la même manière, pour mieux comprendre Greimas, il faudrait lire ses écrits à rebours. C'est justement le cas de l'article "Conditions d'une sémiotique du monde naturel" (ibid.: 49-91), le texte le plus long et sans doute le plus complexe de $\mathrm{Du}$ sens, dont on s'occupera spécifiquement ici. Cet essai se termine en soulignant les difficultés théoriques de l'établissement d'une notation symbolique pour le langage gestuel. Question opératoire, apparemment de détail, mais, d'après Greimas, décisive pour la fondation de la sémiotique en tant que telle:

[...] ce n'est qu'un disposant d'une notation symbolique appropriée [pour le langage gestuel] qu'on pourra penser sérieusement à la constitution d'une sémiotique du monde naturel, condition de la réussite de l'entreprise sémiotique dans son ensemble. (Ibid.: 91)

Pourquoi un enjeu de cette taille? Pourquoi la constitution artificielle d'un métalangage symbolique des gestes peut-elle contribuer à fonder la science de la signification à part entière? Quelle est cette relation si importante entre l'étude du langage gestuel et la constitution de la sémiotique générale? Une brève réponse à ces questions se trouve au début de l'article (ibid.: 50-51), lorsque Greimas propose une "métaphore relevant du discours didactique»: l'analogie entre la découverte de l'écriture et la fondation théorique de la sémiotique générale.

Suivons, pour bien l'éclairer, l'argumentation métaphorique de Greimas. L'invention de l'écriture, reposant sur «une transposition du code utilisant la substance sonore dans un code de type visuel», a présupposé (en amont) «la constitution d'une phonologie implicite nécessaire à une telle transposition» et a provoqué (en aval) «une mutation brusque, qualitative, de la pensée humaine». De la même manière, l'effort pour un dépassement de la linguistique (limitée aux langues naturelles) au profit d'une sémiotique (qui puisse étudier tous les systèmes et les procès de la signification humaine et sociale) «annonce [...] une révolution 
[de la pensée humaine] aussi tâtonnante et aussi difficile». À quelle condition? La réponse se trouve dans le terme caché de la métaphore: à condition que la fondation de la sémiotique générale puisse présupposer à la fois quelque chose comme «la constitution d'une phonologie implicite» - c'est-àdire, en ce qui concerne la gestualité, la possibilité justement d'une transposition d'un code relevant du corps et de la (sa) spatialité dans un code de type visuel - et une notation symbolique adaptée à l'étude des pratiques et des langages gestuels.

La question est relancée plusieurs fois tout le long de l'article: la construction d'une notation symbolique présuppose, par exemple, des choix préalables très nets sur la nature des gestes (unités minimales ou réalités construites), sur les critères de segmentation du syntagme gestuel (et donc sur la segmentation du corps en parties), mais aussi sur la valeur significative du mouvement humain ou sur la distinction entre les attitudes pratiques de l'homme et ses intentionnalités communicatives par le corps. Une question fonctionnelle (visant à une diffusion minimale mais nécessaire du discours scientifique) devient ainsi un problème en même temps théorique (qu'est-ce qu'un geste?) et épistémologique (quelle est la relation entre le sens exprimé par le corps et celui qui découle de l'articulation de la sonorité?). C'est donc l'existence même d'une discipline «nouvelle» comme la sémiotique générale qui est en jeu.

L'enjeu épistémologique ouvert par cet article, qu'on cherchera à décrire patiemment, fait aussi tout son intérêt et toute son actualité. Comme on le verra en conclusion, les positions prises à cette occasion par Greimas constituent autant de réponses anticipées au naturalisme qui semble guider aujourd'hui une partie de la recherche sémiotique et veut penser le monde naturel et le corps comme frontières ou origines du sens.

$$
\text { * * * }
$$

On a vu que l'article "Conditions d'une sémiotique du monde naturel» discute la question capitale de la constitution de la sémiotique générale à partir de la question particulière d'une étude sémiolinguistique des langages dits gestuels. Pour saisir l'enjeu théorique de cet essai, il faut rappeler qu'il s'agit de l'«Introduction" à un numéro de la revue Langages ( $\mathrm{n}^{\circ} 10$, juin 1968) consacré aux "Pratiques et langages gestuels", avec des articles de B. Kochlin ("Techniques corporelles et leur notation symbolique»), J. Kristeva («Le geste, pratique ou communication?»), P. Fabbri ("Considérations sur la proxémique»), F. Rastier ("Comportement et signification "), V. Proca-Ciortea et A. Giurchescu ("Quelques aspects théoriques de l'analyse de la danse populaire»), C. Bremond ("Pour un gestuaire des bandes dessinées»), R. L. Birdwhistell («L'analyse kinésique»), C. Hutt ("Dictionnaire du langage gestuel chez les Trappistes»), R. Cresswell ( Le geste manuel associé au langage»), P. Bouissac ("Volumes sonores et volumes gestuels dans un numéro d'acrobatie»), J. Kristeva et M. Lacoste («Bibliographie»). Cette introduction est reprise deux ans plus tard- avec quelques modifications - dans $D u$ sens. De la même manière que d'autres prologues de Greimas à des ouvrages collectifs ${ }^{1}$, il s'agit d'une introduction écrite à la fin, une sorte de postface qui discute les différents articles de la revue, en proposant une synthèse des questions et une ré-articulation des problématiques posées. Une lecture intégrale de ce numéro de Langages permet donc une meilleure intelligence du texte greimassien qu'on analyse ici (Greimas, 1968).

Le point de départ commun à l'équipe de recherche de Langages est le dépassement d'une étude des gestes considérés comme des unités «naturelles» et, donc, "universelles", c'est-à-dire en deçà de la formation historique des différentes cultures. Comme l'avait déjà soutenu la kinésique américaine (représentée dans la revue par deux extraits de R.L. Birdwhistell et commentée dans le même numéro par l'article de Julia Kristeva), les langages gestuels, comme les langues, n'ont rien de naturel; les gestes sont des produits historiques qui circulent différemment dans chaque culture. Comme les mots, ce ne sont pas des éléments de base, mais des réalités 
construites par des unités distinctives appelées «kinèmes» (eux-mêmes combinaisons des traits minimaux dits «kinés»).

Mais c'est justement la kinésique que discute l'équipe dirigée par Greimas, parce que cette discipline relève d'une épistémologie, implicite, behavioriste et communicationnelle. Pour cette discipline, en fait, le code gestuel se réduit aux «aspects communicatifs du comportement appris et structuré du corps en mouvement" (Kristeva, dans Greimas, 1968: 55). Elle étudie seulement les gestes intentionnellement émis par les différents sujets humains dans des situations communicatives concrètes, en prenant comme modèle analogique la langue verbale et comme méthode d'analyse la linguistique structurale (mais à la lumière d'auteurs très variés comme Darwin, Boas, Sapir, Mead, Whorf, Harris, etc.). Tout ce qui concerne les pratiques humaines - les formes des différentes attitudes, mais aussi les "technologies du corps" (Mauss) - n'est pas l'objet d'analyse de cette discipline (qui donc, en conclut Kristeva, est «subordonnée aux préjugés d'un sociologisme positiviste» [ibid.: 64]). En revanche, une sémiotique structurale de la signification, et non plus de la simple communication, ne peut pas ne pas prendre en considération ces questions.

De la même façon, une autre discipline de recherche qui relève en principe de l'étude sémiotique de la gestualité, la proxémique, révèle des problèmes théoriques et épistémologiques fort similaires. Fondée par l'anthropologue Edward T. Hall, la proxémique étudie la structuration signifiante de l'espace humain en analysant la signification des différentes distances parmi les hommes et leurs orientations réciproques au niveau de micro-espaces (interaction à deux, formes de la territorialité, contraintes de l'ameublement) et de macro-espaces (architecture, urbanisme, paysage, etc.). Comme l'observe Fabbri dans son article, la proxémique présuppose également une épistémologie behavioriste et une sémiologie de l'information. En fait, la «dimension cachée» qui est l'objet d'analyse explicite de cette discipline n'est pas le niveau immanent de la forme signifiante, celui du système sémique de la spatialité, mais, au contraire, le niveau de la surface substantielle de la semiosis, «de la manifestation, de cet événement-communication où s'opère la conjonction du signifiant et du signifié» (Fabbri, dans Greimas, 1968: 73). C'est ainsi que, en reprenant la distinction célèbre de Pike, pour la discipline de Hall «il s'agit de proxetic, pas encore de proxemic» (ibid.: 72) ${ }^{2}$.

L'examen des présupposés épistémologiques (et des effets idéologiques) de ces deux sphères de recherche kinésique et proxémique - pose le problème de la fondation théorique d'une étude sémiotique de la gestualité, c'est-à-dire la question de la définition même de l'objet spécifique de l'analyse, de la délimitation du champ d'observation, de l'individuation des seuils de ce champ et de la reconstruction des rapports de voisinage avec d'autres disciplines. Si on compare, par exemple, l'article de Rastier avec celui de Kristeva, on s'aperçoit que les deux auteurs ont des visions de la gestualité presque opposées, à partir de deux manières différentes de délimiter de façon préliminaire le mouvement corporel signifiant.

Pour Rastier, il faut distinguer entre un comportement humain significatif et un comportement humain non significatif, en posant les conditions préalables selon lesquelles «un comportement peut être considéré comme porteur de signification" (dans Greimas, 1968: 76). Toujours selon lui, et à la suite de Hjelmslev, un comportement est significatif s'il peut être interprété comme le plan d'expression d'un langage (articulé) qui se pose en une relation de présupposition réciproque avec un plan du contenu (à son tour articulé). Étudier la signification du comportement humain veut dire identifier ses unités d'expression et ses unités du contenu, mais aussi, et surtout, leur relation nécessaire. Tout ce qui ne correspond pas à ces caractéristiques n'est pas signifiant, et ne peut être l'objet d'étude d'une sémiotique des gestes. Cette délimitation ne repose ni sur des critères externes au champ d'analyse (communication intentionnelle versus mouvements involontaires du corps; gestes versus pratiques), ni sur des présupposés implicites cachés au moment de 
l'analyse même (signes artificiels versus signes naturels; comportement mythique versus praxis quotidienne), mais sur des conditions scientifiques explicites, communes à tous les langages. Par conséquent, le comportement non significatif «comprend le comportement pratique et le comportement réflexe, c'est-à-dire tous les comportements non symboliques" (ibid.: 81-82).

Pour Kristeva, par contre, la distinction fondamentale est celle entre pratiques, d'un côté, et communication, de l'autre. Le but de son article est de démontrer que la gestualité relève d'une "pensée antinormative» qui «tend à s'évader des grilles de la rationalité "logocentrique" "(dans Greimas, 1968: 48). La tradition philosophique occidentale, selon Kristeva, a toujours privilégié la relation son/idée, en soulignant à plusieurs reprises le primat du langage verbal et en présentant par conséquent «toute gestualité comme mécanique, redondante par rapport à la voix» (ibid.: 49). L'enjeu d'une sémiotique générale visant à considérer le rôle des gestes dans l'action humaine signifiante serait, au contraire, de sortir de cette tradition, en affirmant l'importance fondamentale de la production, de la praxis, pour la constitution des cultures. Au-delà de ces questions philosophiques plus générales, cette approche comporte des conséquences théoriques d'une certaine importance: l'idée d'une «irréductibilité du geste au langage verbal» (ce qui est en opposition avec la tradition pensant les gestes comme simple support de la verbalité) et celle, encore plus fondamentale, d'un élargissement de «la notion même du langage, compris non plus comme communication, mais comme production" (ibid.: 50) (s'opposant à la vision fonctionnelle des langues comme de simples instruments pour la transmission des informations). Loin d'être considérée comme un accompagnement accessoire de la communication verbale, la gestualité acquiert le statut d'une «activité antérieure au message représenté et représentable», un travail qui précède la constitution même du signe et des mots, une activité qui fonde la possibilité même de désignation propre aux mots. Bref, toute gestualité est une pratique.
**

Pour essayer de résoudre ces questions et d'autres problèmes plus spécifiques qui en découlent, Greimas énonce les conditions théoriques, non simplement d'une sémiotique des gestes, mais, plus généralement, d'une sémiotique du monde naturel. Tous les gestes, selon une tradition philosophique très ancienne (qui va d'Aristote à Wittgenstein, en passant par les Idéologues), relèvent d'une semiosis qui est de l'ordre non pas de l'«arbitraire» ou du «conventionnel» linguistique, mais d'une sorte d' "analogie», de «motivation" et, donc, de «nature». Mais qu'est-ce que le «monde naturel» pour Greimas? Quelle valeur donner à cette notion fort ambiguë et à des notions limitrophes comme «signe naturel» (versus «signe artificiel»), «référent» (versus «signifié»), «choses» (versus «mots»), "nature» (versus «culture»)?

En suivant l'interprétation hjelmslévienne de la notion d'arbitraire du signe formulée par Saussure (Hjelmslev, 1957), Greimas postule que l'arbitraire ne concerne pas tant la relation entre signifiant et signifié, ou si l'on veut entre expression et contenu, que celle entre forme et substance (de l'expression et du contenu). La langue «est une forme - ou mieux, l'enchevêtrement de deux formes - indifférente à la substance dans laquelle elle se trouve manifestée» (Greimas, 1970: 49). Cela comporte des conséquences théoriques très précises et fondamentales: si la substance est une variable et la forme une constante, toutes les substances (en tant que formées sémiotiquement) peuvent être chargées de manifester des expressions et des contenus; pas seulement la substance sonore (qui se manifeste dans l'oralité) ou la substance graphique (qui se manifeste dans l'écriture), mais toutes les substances du monde, c'est-à-dire toutes les façons sensibles dont le monde se révèle à nous par notre appareil sensoriel (par notre corps): qu'elles soient visuelles, tactiles, sonores, olfactives, sensorimotrices, etc.

C'est ainsi que la linguistique des langues verbales ne peut pas ne pas s'élargir en une sémiotique de tous les systèmes et les procès de signification, en devenant 
une sémiotique du monde naturel. Et c'est ainsi que le «monde naturel» devient, pour le sémioticien, un ensemble très varié de manifestations sensorielles (des substances) acquérant du sens, pour l'homme, par les différents modes de leur articulation formelle. En d'autres mots, le monde est «naturel» de la même manière que les langues dites naturelles: il s'agit de constructions culturelles par lesquelles des formes d'expression se posent en présupposition réciproque avec des formes de contenu. La différence entre les langues («naturelles») et le monde ("naturel») est une question de substance, non de forme; une question donc indifférente à la production, culturellement et historiquement déterminée, de la signification humaine. «Naturel» veut donc dire, en ce contexte théorique, quelque chose comme "habituel», « déjà donné par le sens commun " ${ }^{3}$, quelque chose que l'individu retrouve au moment de son appréhension subjective de ce qui l'entoure ${ }^{4}$. Comme on lira dans l'entrée «Monde naturel» du Dictionnaire de Greimas et Courtés:

Nous entendons par monde naturel le paraitre selon lequel

l'univers se présente à l'homme comme un ensemble de qualités sensibles, doté d'une certaine organisation qui le fait parfois désigner comme «le monde du sens commun». Par rapport à la structure "profonde" de l'univers, qui est d'ordre physique, chimique, biologique, etc., le monde naturel correspond, pour ainsi dire, à sa structure «de surface»; c'est, d'autre part, une structure «discursive», car il se présente dans le cadre de la relation sujet/objet, il est «l'énoncé» construit par le sujet humain et déchiffrable par lui. (1979: 233)

En reprenant certains passages de la philosophie phénoménologique de M. Merleau-Ponty et de l'anthropologie structurale de C. Lévi-Strauss, et en accord avec les propositions sur la typologie des cultures avancées dans les mêmes années par I.Lotman, Greimas propose de penser la différence entre nature et culture comme une construction humaine différente selon les cultures, une délimitation orientée entre ce qui est dans chaque culture et ce qui ne l'est pas, qui reste au dehors d'elle. En utilisant les catégories explicatives du carré sémiotique (proposées aussi dans $D u$ sens), on pourrait dire que, avant d'être une Nature, cette entité doit passer par le stade de la Non-Culture (d'une négation qui précède, comme toujours, l'affirmation). Nature et Culture, sortes d'universaux sémantiques collectifs, construisent une opposition de base à l'intérieur de chaque culture, variable dans l'espace et dans le temps, par laquelle chaque culture peut articuler ses signes, ses langages, ses attitudes signifiantes, ses codes 5 . certaine importance, aussi bien sur le plan théorique que sur le plan méthodologique. En première instance, cette conception de la sémiotique (entendue, suivant Hjelmslev, comme un élargissement nécessaire de la linguistique structurale, et non comme un simple geste de curiosité intellectuelle) consiste en un dépassement radical des hypothèses sur la "spécificité" des langages formulées par la sémiotique américaine (voir Morris) et par la sémiologie européenne de la communication (voir Buyssens). Selon ces théories, les systèmes des signes se différencient sur la base de canaux de transmission, c'est-à-dire de substances qu'ils utilisent sur leur plan de l'expression: substance sonore pour les langues verbales, substance visuelle pour les images, substance audiovisuelle pour le cinéma et la télévision, substance somatique pour les gestes, etc. Mais, si la signification dépend de la forme et est indifférente à la substance, c'est l'articulation formelle interne aux langages qui peut les différencier, non la substance par laquelle ils se manifestent à nous. Ainsi, dans cet article, Greimas pourra dire que les différences principales entre la langue verbale et le langage gestuel ne concernent pas leurs substances, mais peuvent être établies en utilisant la distinction hjelmslévienne entre signes et symboles (et, comme il dira ultérieurement, semi-symboles ${ }^{6}$ ) ou celle, élaborée par Benveniste, entre énoncé et énonciation. En d'autres mots, elles peuvent être établies par des modèles tout à fait internes aux faits linguistiques et sémiotiques. 
De la même manière, la nouvelle formulation de la problématique sémiotique proposée par Greimas permet de redéfinir la notion de référent, introduite par le néo-positivisme logique (et utilisée dans la définition morrissienne du signe) mais tout à fait refusée par la linguistique et la sémiologie structuraliste depuis Saussure jusqu'à Barthes. Selon Greimas, le problème de la «chose» externe au langage, à laquelle le langage peut (ou doit) faire référence, existe seulement si l'on considère comme objet exclusif de l'analyse linguistique le langage verbal, si l'on considère un seul type de système de signification: celui des langues naturelles. Mais si le monde naturel (interprété dans le sens très précis qu'on a reconstruit plus haut) devient aussi un objet d'étude sémiolinguistique, si donc ce monde "extralinguistique" doit être considéré "comme le lieu de la manifestation du sensible, susceptible d'être la manifestation du sens humain» (Greimas, 1970: 52), il ne peut plus être interprété comme un référent absolu externe aux langues, mais doit l'être comme un autre langage, ou mieux: comme un ensemble d'autres langages, d'autres signes, d'autres systèmes et procès de signification. À ce propos, on lit encore dans l'entrée «Référent» du Dictionnaire:

Le monde extralinguistique, celui $d u$ "sens commun», est informé par l'homme et institué par lui en signification [...] tel monde, loin d'être le référent [...] est, au contraire, lui-même un langage biplanaire, une sémiotique naturelle [...]. Le problème $d u$ référent n'est alors qu'une question de corrélation entre deux sémiotiques [...], un problème d'inter-sémioticité.

(Greimas et Courtés, 1979: 312)

L'évidence du monde de la phénoménologie est le lieu où se constituent l'expérience et la conscience humaines; toutefois, le monde possède également des articulations internes qu'il faut aussi reconstruire et expliquer. Ainsi, le «référent» n'est plus à considérer comme une donnée empirique de base qui peut être représentée plus ou moins scrupuleusement par des signes construits ad hoc (selon la dictée néopositiviste), comme une construction des langues (selon la théorie de Sapir et Whorf), ou comme une entité indépendante de l'immanence linguistique (selon le structuralisme linguistique). La relation entre la langue naturelle et le monde naturel, considérée de ce point de vue, n'est plus une relation de référence entre un système de signes et un ensemble de nonsignes, mais une corrélation de deux systèmes de signes (ou plus), une transformation sémiotique; en somme: une traduction.

On comprend alors les raisons de certaines affirmations de l'introduction générale à $D u$ sens: la constitution des signes, des langues, des systèmes de signification n'est jamais la métamorphose mystérieuse d'une réalité non signifiante, dite "pure», «blanche», etc., en des réalités significatives. Bien au contraire, chaque construction du sens est toujours un passage d'un signe à l'autre, une transformation orientée: le sens est renvoi, mais aussi direction.

L'homme vit dans un monde signifiant. Pour lui, le problème du

sens ne se pose pas, il s'impose comme une évidence, comme un "sentiment de comprendre» tout naturel. Dans un univers "blanc» où le langage serait pure dénotation des choses et des gestes, il ne serait pas possible de s'interroger sur le sens: toute interrogation est métalinguistique. (Greimas, 1970: 12-13)

Le problème n'est donc pas celui du sens, mais celui de ses conditions d'articulation, des procès de constitution de la signification:

La signification n'est donc que cette transposition d'un niveau de langage dans un autre, dans un langage différent, et le sens n'est que cette possibilité de transcodage. (Ibid.: 13)

Il ne s'agit pas d'idées totalement originales. LéviStrauss, dans ses réflexions sur l'arbitraire du signe, avait tenu des propos similaires. La signification des cultures, comme disait Saussure, n'est pas produite par des motivations naturelles. En revanche, elle se constitue à partir de significations précédentes qui, par bricolage, contribuent subrepticement à la construction des codes sociaux. Prenons l'exemple très simple du feu vert: s'il est tout à fait arbitraire que «les règlements de la circulation ont assigné leurs valeurs sémantiques respectives au feu rouge et au feu vert», il est également vrai que ce choix se fonde sur le fait que, dans notre 
culture, «le rouge évoque le danger, la violence, le sang; et le vert, l'espoir, le calme et le déroulement placide d'un processus naturel comme celui de la végétation ". Mais si l'on veut modifier le système, en renversant la signification réciproque de ces deux couleurs, «sans doute le rouge serait-il perçu comme témoignage de chaleur humaine et de communicabilité, le vert comme symbole glaçant et venimeux" (Lévi-Strauss, 1958: 108). Il existe donc une «symbolique traditionnelle» qui est une sorte de réserve de matériaux déjà signifiants qu'une culture peut utiliser pour la production de significations nouvelles (qui ne sont donc pas tout à fait «nouvelles", mais en quelque sorte pré-contraintes ${ }^{7}$ ). Une fois cette signification activée, il est possible d'établir, a posteriori, la symbolique «naturelle» qui a été utilisée et celle qui ne l'a pas été ${ }^{8}$.

On comprend pourquoi la notion greimassienne de «monde naturel» découle justement d'une enquête sur la gestualité. Les gestes, au moment où se bâtissent des systèmes de communication parmi les hommes, articulent les parties et les mouvements du corps dans un espace qui l'entoure. Mais la sélection de ces parties et de ces mouvements (en tant que substances d'expression de la communication gestuelle) n'est pas totalement autonome: elle se base, précisément comme le feu vert de Lévi-Strauss, sur des morceaux de significations préexistantes, sur des signes déjà faits, sur des figures chargées de sens possibles. Par exemple, l'opposition lourd/léger, que la danse utilise pour produire ses significations, a déjà des valeurs dans le monde naturel, la légèreté étant euphorique et la lourdeur dysphorique (ou vice versa). Et la même chose vaut pour d'autres oppositions possibles comme stase/mouvement, action/procès, inchoatif/ terminatif, haut/bas, prospectif/rétrospectif, etc., qui, une fois utilisées dans des codes de communication gestuelle, sont resémantisées.

Pour cette raison, dit Greimas, il ne faut pas confondre la praxis somatique (qui est une affaire de pratiques énoncées par des sujets d'actions, des corps en mouvement) avec la communication gestuelle (qui est, au contraire, un fait d'énonciation produit par des corps-émetteurs pour des destinataires qui l'interprètent). Dans le premier cas, c'est un problème de sens comme direction: la signification des actions corporelles est produite par un sujet qui a ces «programmes stratégiques» (analogues aux "programmes phonatoires» qui transforment la substance sonore en forme de l'expression linguistique - et aux "programmes narratifs» dont on parlera quelques années plus tard), comme faire un nœud à sa cravate ou travailler dans une usine. Dans le deuxième cas, c'est un problème de sens comme renvoi: la communication se produit à partir d'un destinateur qui - plus ou moins intentionnellement transmet des messages à un destinataire, comme dans le cas de la gesticulation sémaphorique artificielle ("où une langue naturelle est sous-tendue en tant que code du contenu référentiel» [Greimas, 1970: 63]). Le problème n'est plus de conserver la distinction entre pratiques et gestes, entre des attitudes non significatives et une véritable communication gestuelle, comme la discussion à distance entre Kristeva et Rastier. Au contraire, pour Greimas, il faut remarquer que le corps peut signifier à deux niveaux différents, celui de l'énoncé et celui de l'énonciation:

[...] dans la praxis gestuelle, l'homme est sujet de l'énoncé, tout en étant un «il» pour nous, il est le «je» agent de l'énoncé, le sujet des fonctions qui constituent son comportement; dans la gestualité communicative, l'homme est le sujet de l'énonciation: il est un «tu» pour nous, mais un «je» pour lui-même, dans la mesure où il cherche désespérément à produire et à transmettre des énoncés. 9

* *

De ces conditions théoriques de base pour une sémiotique du monde naturel découle la possibilité d'une étude scientifique des gestes - que toutefois la recherche sémiotique subséquente n'a plus repris véritablement ${ }^{10}$. La valeur de l'essai greimassien dont on a cherché à rétablir ici les argumentations explicites et implicites est donc en première instance de type historique, en tant que moment fondamental pour l'agencement subséquent de la sémiolinguistique structurale et générative greimassienne et post- 
greimassienne. On a vu que réside en ces pages une configuration cohérente des traits épistémologiques de la sémiotique générale (présupposition réciproque de l'expression et du contenu; primauté de la forme sur la substance; critique des notions de spécificité des codes et de référent). Et l'on pourrait aussi y retrouver la préfiguration d'un certain nombre d'idées que la recherche postérieure développera (la générativité, la textualité, le semi-symbolisme, la figurativité...), ainsi que des positions théoriques qui seront abandonnées dans les années suivantes (l'hétéronomie des gestes, la démarche componentielle...).

Mais la lecture, ou la relecture, de ces pages peut être utile surtout pour les recherches actuelles en sémiotique, où il est question en même temps des faits sociaux et des corps, des enchaînements collectifs d'énonciation et de perception sensorielle, des formations culturelles et des constitutions des subjectivités, des pratiques sociales et d'instances présubjectives. Au-delà des oppositions un peu naïves entre sémiotiques générative et interprétative, objectale et subjectale, tensive et sociale, textuelle et culturelle, du continu et du discontinu, de l'immanence et de l'expérience, etc., il s'agit toujours de travailler sur les mêmes problèmes fondamentaux: ceux qui ont le sens comme domaine commun d'investigation et la signification comme visée unitaire d'une quête, sinon scientifique, du moins intellectuelle.

À ce propos, l'article de Greimas sur le monde naturel peut servir d'orientation, en négatif, pour ainsi dire, contre certaines orientations actuelles de la recherche en signification. Il prend position, avant la lettre, contre toute forme de naturalisme qui, de façon plus ou moins évidente, veut s'imposer dans la théorie de la signification. Il n'y a rien de naturel dans les langues dites «naturelles» et, on l'a vu, il n'y a rien de naturel non plus dans le "monde naturel", monde du sens commun articulé culturellement, structure de surface d'un monde physique ou chimique profond sur lequel le sémioticien n'a rien à dire. Si le naturalisme domine aujourd'hui la plupart des programmes de recherche en sciences sociales, $y$ compris certaines formes de sémiotique, la leçon que
Greimas nous a laissée est pourtant très claire: le sens est déjà là, avant même sa saisie humaineet sociale; le problème de chaque culture, de chaque société, de chaque discours est de le mettre en condition de signifier, de l'articuler en même temps dans les deux plans nécessaires de l'expression et du contenu.

Articuler le sens, le mettre en condition de signifier ne veut toutefois pas dire le transposer dans quelque autre langage ou code, mais bien le traduire par quelques autres systèmes de signification. C'est pourquoi ces pages de Greimas nous permettent de nous opposer à toutes les hypothèses théoriques portant sur la "genèse» du sens - aujourd'hui très répandues. Il ne faut pas confondre, on le sait, générativité et genèse: si l'on peut parler des différents niveaux de signification (plus ou moins profonds, plus ou moins abstraits), cela ne signifie pas que le "profond" soit à l'origine (temporelle aussi bien que logique) du sens; mais plutôt que l'on doive prévoir des pertinences différentes pour le saisir. Selon Greimas, le problème de l'origine ne se pose pas: chaque interrogation humaine du monde est toujours métalinguistique (ou métasémiotique), et comprendre, c'est transposer, re-dire, reconstruire par d'autres signes ${ }^{11}$.

D'où une troisième précieuse indication en négatif: toutes les hypothèses qui essaient de retrouver dans le corps humain le lieu fondamental où se constitue un sens dit «auroral», l'équivalent des premiers vagissements signifiants de l'homme, n'ont pas de fondement épistémologique. Le corps ne s'interpose pas entre le désarticulé et l'articulé, le continu et le discret, le sens et la signification: il est toujours déjà dans la signification. Il n'y a pas d'ouverture originaire de la subjectivité du corps, suivie de transformations plus ou moins contraignantes réglées par la fonctionnalisation sociale. Prendre le corps en considération, c'est déjà le penser comme un élément ou un procès social, ayant un destin culturel tracé par des valeurs et des intérêts précis, en contribuant à les former ou à les déconstruire. Si l'on dit que la subjectivité humaine dispose d'une «base corporelle», cela ne signifie pas 
qu'elle se fonde sur une «nature» comme condition de possibilité universelle des transformations successives - individuelles et collectives. Cela veut dire, au contraire, que le sujet se constitue et se reconstitue en allant d'expériences pré-subjectives aux instances intersubjectives, et vice versa, toutes les deux étant d'origine somatique. Entre subjectivité et corporalité, il n'y a pas d'adhérence absolue et constante, mais un contact partiel et temporaire. Il y a là une dislocation constitutive qui crée, et en même temps affaiblit, l'identité des individus et des groupes sociaux. Je suis mon corps, mais en même temps j'ai un corps, dans lequel je me reconnais en partie, mais auquel je ne délègue pas la responsabilité entière de mon existence et de mes expériences ${ }^{12}$.

\section{N O TES}

1. Par exemple celui sur la poéticité de 1972 ou celui, jamais publié, sur la visualité de 1984.

2. En tout cas, remarque Fabbri en conclusion de son article, le champ d'étude de la proxémique se révèle fort important parce qu'il «[...] enrichit la notion même de geste. Par une implication double, le geste de l'autre délimite de l'extérieur ce que le schéma proxémique projette hors de mon corps en réglant l'espace de mon propre geste. On parle et on est parlé par l'autre. D'un effet semblable au phénomène physique dit de la capitation, le langage opère sur la substance spatiale - de la même manière que les ultrasons sur les liquides - créant le vide, la différence, l'espacement, donc la relation et le sens " (dans Greimas, 1968: 75). Mais cette remarque ne sera pas reprise par Greimas dans son essai introductif.

3. Il faut rappeler à ce propos que le chapitre de Du sens qui suit celui discuté ici s'appelle justement "Pour une sociologie du sens commun". Dans cet essai, Greimas articule la notion hjelmslévienne de "connotation" en vue d'une nouvelle orientation de la recherche en signification qui sera, avec les travaux d'E. Landowski (1989) et d'autres, la sociosémiotique. On lit, en fait, que «l'analyse des systèmes connotatifs" mène à la constitution d' "un domaine de recherches autonome" qui "permet d'intégrer dans la recherche sémiotique [...] un champ de significations dont l'appréhension scientifique parait encore impossible et qu'on invoque souvent comme le niveau du vécu et du senti, du quotidien et de l'humain pour l'opposer au caractère abstrait et décharné de la sémiotique" (Greimas, 1970: 99-100).

4. Au fond, c'est l'idée philosophique de la "pré-compréhension", point d'arrivée de l'herméneutique et point de départ de la sémiotique. Pour la science de la signification, il s'agit non pas de montrer les difficultés théorétiques de l'idée gnoséologique de "représentation ", mais de rétablir les mécanismes de sa construction discursive, un «effet de représentation». Le travail de Barthes (1957), qui parlait de naturalisation, est à ce propos fondamental.

5. Voir Greimas et Courtés (1979), entrées «Nature», «Culture» et "Univers".

6. La notion de «langage semi-symbolique" est développée dans Greimas (1984); mais on peut la retrouver in nuce dans Greimas (1972), ainsi que dans l'essai étudié ici, lorsqu'on parle d'une corrélation «à la fois arbitraire et constante», «d'une catégorie phémique du plan de l'expression avec une catégorie sémique du plan du contenu " (Greimas, 1970 : 72). Ce n'est pas un hasard alors si, dans l'article paru en 1984, Greimas essaiera d'expliquer le semi-symbolisme et utilisera comme exemple, justement, le système mimique de l'affirmation et de la négation reconstruit par Jakobson (1972) - plusieurs fois cité déjà dans l'essai sur la gestualité.

7. L'idée du «bricolage» sera développée par cet auteur dans La Pensée sauvage (Lévi-Strauss, 1962) et sera réutilisée par Floch (1995) pour expliquer la notion de "praxis énonciative».

8. Toute la théorie subséquente de la figurativité, qui souvent tire son origine de Bachelard, n'est donc qu'un déploiement de cette remarque lévi-straussienne.

9. Greimas, 1970: 67. De cette idée dérive, selon Greimas, «la pauvreté de ce qu'on appelle le langage gestuel», qui «semble provenir de cette impossibilité d'un syncrétisme entre le sujet de l'énonciation et le sujet de l'énoncé. Le code de la communication gestuelle ne permettant pas de construire des énoncés, et celui de la praxis gestuelle ne manifestant le sujet que comme sujet du faire, il n'est pas étonnant que les codes visuels artificiels, pour s'ériger en langages, soient des constructions composites, où les éléments constitutifs d'énoncés sont obtenus par des procédés de description imitative». Mais, il est évident que la notion d'énonciation utilisée ici par Greimas est encore naïve: elle n'est pas celle de Benveniste, c'est-à-dire celle qui reconstruit les marques de l'intersubjectivité à l'intérieur de la langue. Pour un développement théorique de cette distinction entre corps énoncé et corps énonçant, voir Fontanille (2004, chap. 5).

10. Pour un travail sémiotique sur la praxis gestuelle, voir Floch (1990, chap. 2), qui étudie le comportement des voyageurs du métro de Paris. 11. Voir Fabbri $(1998,2001)$, qui a posé de manière très précise la traduction au cœur même de la signification.

12. Pour une discussion plus approfondie de ces thèmes, voir Marrone (2001, 2006).

\section{RÉFÉREN CES BIBLIO G RAPH IO U ES}

BARTHES, R. [1957]: Mythologies, Paris, Seuil.

FABBRI, P. [1998]: La Svolta Semiotica, Rome-Bari, Laterza; [2001]: Elogio di Babele, Rome, Meltemi.

FLOCH, J.-M. [1990], Sémiotique, marketing, communication, Paris, PUF; [1995]: Identités visuelles, Paris, PUF.

FonTANILLE, J. [2004]: Soma et séma. Figures du corps, Paris,

Maisonneuve \& Larose.

GreImAs, A.J. [1970]: Du sens, Paris, Seuil;

__ [1972]: «Introduction», Essais de sémiotique poétique, Paris, Larousse; [1976]: Maupassant. La sémiotique du texte. Exercices pratiques,

Paris, Seuil;

[1984]: «Sémiotique figurative et sémiotique plastique», Actes

sémiotiques. Documents, vol.IV, n ${ }^{\circ}$ 60, 5-24;

(dir.) [1968]: « Pratiques et langages gestuels», Langages, n 10, juin.

Greimas A. J. et J. COURTÉs [1979]: Sémiotique. Dictionnaire raisonné de la théorie du langage, tome I, Paris, Hachette.

HJelmsLEV, L. [(1957) 1959]: «Pour une sémantique structurale»,

Travaux du Cercle linguistique de Copenhague, vol. XII, no 59, 96-112.

JAKOBSON, R. [1972]: "Motor Signs for "Yes" and "No" ", Language in Society, $\mathrm{n}^{\circ} 1,91-96$

LANDOWSKI, E. [1989]: La Société réfléchie. Essais de sociosémiotique,

Paris, Seuil.

LÉVI-STRAUSS, C. [1958]: Anthropologie structurale, Paris, Plon; [1962]: La Pensée sauvage, Paris, Plon.

Marrone, G. [2001]: Corpi sociali. Processi comunicativi e semiotica del testo, Turin, Einaudi;

[2006]: Le Traitement Ludovico. Corps et musique dans l'Orange mécanique, Limoges, Presses universitaires de Limoges. 